\title{
Lasing Due To The External Field And Target's Permeability And Conductivity
}

\author{
Abdelaziz Hagar Abdelrahman ${ }^{*}$ \\ "Physics Department, Collage of Science\& Arts EL-Mithnab, Gassim University, KSA.
}

\begin{abstract}
In this work, Maxwell equations used to find the effect of polarity and conductivity effect probability of electromagnetic wave when passing the matter. The wavelength and intensity of the incident electromagnetic wave decrease in side the matter due to polarity and conductivity of the matter. We find that, part of the intensity loss as electric current inside the matter. In addition, lasing can be done due to electric oscillate between electrons and nuclei in an atoms when, the electromagnetic wave passing the matter. In this research also, the conditions of occurrence of the emission of a laser requires that depends on the wavelength and the permeability and conductivity was found.
\end{abstract}

Keywords: Maxwell equations, wavelength, photons, lasing, pointing vector.

\section{Introduction}

The history of the laser starts since 1917 when Einstein discovered a new mechanism by which electromagnetic (E. M) radiation interacts with matter [1-6]. This mechanism is now known as stimulated emission of E. M radiation. In this process, an incident photon on an atom forces it to emit a photon in phase and in the same direction [3]. Nearly, all lasers produced as result of electrons jumping from an excited energy level within a radiating species to a lowerlying energy level and, in the process, radiating light that contributes to the laser beam [7]. If an electric field $E(t, x)$ is applied to a conducting medium, a current density $j(t, x)$ will be produced in this medium.[8]. Laser technology plays an important role in our day life. Beside its application in telecommunication and quantum computer, laser is now used in medicine [3].

The foundation of this new Maxwell equation requires finding the energy density exchange in medium [9]. Relation between electrical field (E) and magnetic field (B) in the electromagnetic (E.M) waves that propagated vertical and horizontal on a slap this was done in sections (2). Section (3) is devoted to exchanges energy and momentum with the medium. Definition of height of pointing vector discussed in section (4). The new lasing equation based on the E.M wave interaction with a medium was exhibited in section (5).

\section{The Electromagnetic Waves}

Light consists of electromagnetic waves moving through space or medium $[9,10,11]$. These waves consist of variation in two types of fields in space. The two types of fields are electric field $\mathrm{E}$ always oscillates vertically to from an electric wave and the magnetic field B always oscillates horizontally, to from a magnetic wave [12]. The two fields are perpendicular to each other and to the direction of propagation of the wave. All electromagnetic wave have the same basic composition but they differ only in frequency and wavelength [13].

\section{The Propagation Wave}

E.M waves were defined to be "self-sustaining oscillation of the electromagnetic field [13-14]. To be self-sustaining, they take on the form of transverse waves, and are the product of two concepts: the electric field and the magnetic field. Michael Faraday is discovered in 1831 that by having a changing magnet field around a conductor, one could produce an electrical current.[3] However, there must be some sort of force to act upon the charge carriers in the conductor. Therefore, we can infer that in order for there to be an induced current, there must be an induced electric field to cause the current. Later, in 1855, James Clerk Maxwell hypothesized that by having a changing electric field, there was an induced magnetic field.[9,13]. He then went further with his hypothesis, stating that it would be possible, supposing that a changing of each one causes another. That wave could be created that was self-sustaining, provided that both the field were perpendicular to each other, and also perpendicular to the direction in which the wave is traveling.[13].

All light consists of wave variation in electric and magnetic field. Many applications require a particular direction of the electric field and magnetic field. Polarization of light describes the orientation of electric field in a space $[14,15,16]$.

In this section, one tries to see how the electromagnetic field respond the properties of the medium. To see how the medium change the frequency and the energy of electromagnetic waves, we can write the wave $[17,18]$. The electrical field (E) one can written as; 
$E=E_{m} e^{i(k x-\omega t)}$

Thus, the $\mathrm{E}$ and magnetic field (B) defined as sign wave

$E=E_{m} \sin (k x-\omega t)=E_{m} \sin (2 \pi \nu+\varphi)$

$B=B_{m} \sin (k x-\omega t)=B_{m} \sin (2 \pi v t+\varphi)$

From Faraday equation, $\oint E d S=-\frac{d \phi_{b}}{d t}$ we find that $\mathrm{B}$ decrease when $\mathrm{t}$ increase, to cover this $\mathrm{E}$ increase with distance [19].

$\oint E d S=(E+d E) h-E h=h d E$

The magnetic flox $\left(\phi_{B}\right)$ written as;

$\phi_{B}=(B)(d x h)$

$\oint E d S=-\frac{d \phi_{b}}{d t}=\frac{(B)(d x h)}{d t}=d h \frac{d B}{d t}$

From eq. (4) and (6)

$h d E=-d h \frac{d B}{d t} \Rightarrow \frac{d E}{d x}=-\frac{d B}{d t}$

Used eqs.(2 and 3) in eq.(7) one can get;

$$
k E_{m} \cos (k x-\omega t)=\omega B_{m} \cos (k x-\omega t) \Rightarrow \frac{E_{m}}{B_{m}}=\frac{\omega}{k}=c \Rightarrow E_{m}=c B_{m}
$$

Eq.(8) the magnetic field is too less compare with the electric one. When used horizontal of a slap the opposite case seen, therefore, B greater than E, one can see that clearly in Ampere low as ;

$$
\begin{aligned}
& \oint B d S=\varepsilon_{0} \mu_{0} \frac{d \phi_{E}}{d t}=\frac{(E)(d x h)}{d t}=d h \frac{d E}{d t} \\
& -(B+d B) h+B h=-h d B
\end{aligned}
$$

From eq. (9) and (10)

$$
-h d B=-d h \frac{d E}{d t} \Rightarrow \frac{d B}{d x}=-\varepsilon_{0} \mu_{0} \frac{d E}{d t}
$$

Used eq.s (2 and 3$)$ in eq.(11) one can get;

$$
-k B_{m} \cos (k x-\omega t)=\omega E_{m} \cos (k x-\omega t) \Rightarrow \frac{E_{m}}{B_{m}}=\frac{k}{\varepsilon_{0} \mu_{0} \omega}=\frac{k}{\omega} \frac{1}{\varepsilon_{0} \mu_{0}}
$$

$$
E_{m}=\frac{1}{c} \frac{1}{\varepsilon_{0} \mu_{0}} B_{m}
$$

From eq. (8) and (13)

$c=\frac{1}{\sqrt{\varepsilon_{0} \mu_{0}}}$

\section{Pointing Vector Theorem}

We need to quantify the associated power flow. We easily obtain a hint of how to calculate power flow by recalling our circuit theory, where, Power equal to potential voltage divided by current flow through the conductor, or by a dimensional analysis of the fields. [3,9,17].

Remember that the units of $\mathrm{H}$ are (Amp/meter) and the units of $\mathrm{E}$ are $(\mathrm{V} / \mathrm{m})$. Their product (Amp Volts/m2) has units of (Watts/area), which is a power density, just what we want. The product must clearly have a direction associated with it, and it ought to somehow point in a reasonable direction. A reasonable direction for power flow in lossless, or magnitude, homogeneous, linear media, such as free space, would be in the direction of propagation. We might therefore, guess that EXH would be a reasonable definition of power density.

We will consider the complex Pointing vector for time harmonic plane electromagnetic waves in phasor notation. This requires some thought because of the nonlinear nature of EXH. As the wave travels, it 
carries this energy along with it. The energy flux density (energy per unit area, per unit time) transported by the fields is given by the pointing vector.[20].

$\vec{S}=\vec{E} x \vec{H}$

Electric and magnetic (B) field are orthogonal (perpendicular, Right Hand Rule), in-phase, and the ratio of the field magnitudes is the impedance. It is very useful to express this ratio of electric and magnetic fields in terms of $\mathrm{H}$, rather than $\mathrm{B}$.

$\mu_{0} \vec{H}_{y}=\frac{\vec{E}_{x}}{c}=\sqrt{\varepsilon_{0} \mu_{0}} \vec{E}_{x}$

One can found that, the ratio of $\mathrm{H}$ to $\mathrm{E}$ is the intrinsic wave impedance, $\eta 0$, after rearranging eq. (16);

$\frac{\vec{E}_{x}}{\vec{H}_{y}}=\mu_{0} c=\sqrt{\mu_{0} / \varepsilon_{0}} \equiv \eta_{0}$

To find the height of $S$ that acts as energy pass through a surface (A) per unit time (t). As S,E,B were moment value one can get;

$$
\vec{S}=\frac{1}{\mu_{0}} E B
$$

From eq. (8) and (18) one can get;

$$
\vec{S}=\frac{1}{\mu_{0} c} E^{2}=\frac{1}{\mu_{0}} c B^{2}
$$

The total energy flux (U) calculate by summations of electric and magnetic field flux $\left(U_{E}\right)$ and $\left(U_{B}\right)$, respectively, in the form;

$U=U_{E}+U_{B}=\frac{1}{2} \varepsilon_{0} E^{2}=\frac{1}{2 \mu_{0}} c B^{2}=\frac{1}{2}\left(\varepsilon_{0} E^{2}+\frac{B^{2}}{\mu_{0}}\right)$

Used eq. (14) in eq.(20) we found ;

$U==\frac{1}{2 c} S+\frac{1}{2 c} S=\frac{S}{c}$

From eq. (20) and eq. (21) for volume has very small thickness $d x \rightarrow 0$ one can get;

$U_{E}=U_{B}$

Instead of describing the electromagnetic (E.M) when interaction with matter in terms of a 'static vectorial', it turns out that it is for most purposes more useful to introduce the concept of a field and to describe the E.M interaction in terms of an electrostatic force $(\mathrm{F})$ action oscillate in medium at a distance (x). Electric field (E) oscillate in space with magnetic field (B) which, are perpendicular to the propagation direction (z). Radiation matter depends on E, so to get action of interaction E useful. If the electromagnetic pass a surface (A), thickness $d x>>0$ in this case to get pointing vector we can used:

$d t=\frac{d x}{c}$

From eq. (21) and eq. (23) the change of the energy flux (U) due to the change of the thickness of the volume can be get out among to the;

$d U=U d V=\frac{S}{c} A \cdot d x=\frac{S}{c} A c d t=S A d t$

In addition, the pointing vector equal to;

$S=\frac{1}{A} \frac{d U}{d t}$

The rate of energy flux $\left(\frac{d U}{d t}\right)$, is define of the power of the electromagnetic wave pass which, through the volume, so one can rewrite $\mathrm{S}$ as;

$S=\frac{P}{A}$ 
From eq. (26), the height of pointing vector defined as; rate of power per unit surface.

Intensity of Electromagnetic Wave

$$
I=\vec{S}_{d V}=\frac{1}{\mu_{0} c}\left(E^{2}\right)_{d V}=\frac{1}{\mu_{0} c} E_{m}{ }^{2}\left(\sin ^{2}(k x-\omega t)\right)_{d V}
$$

Usually if the average time period greater than the period of one session, valuable this case we can neglect the influence of fractures session and put the $\sin 2$ equal to their average $1 / 2$. We found;

$I=\frac{1}{\mu_{0} c} E_{r m s}^{2}=\frac{1}{\mu_{0}} E_{r m s} B_{r m s}$

By comparing eq. (28) with eq. (25) the Erms act as electromagnetic wave have amplitude $E_{\text {rms }} B_{\text {rms }}$, this case give us the intensity at the point at the center of the source.

If the propagated waves did not loss part of energy that means, the energy acts as circular wave, at point on wave front get from generation source. The rate of energy at any point of propagation wave that, pass circular surface depend on the radius bool when the point source at the center. The energy density at any point surrounding the source equal to;

$$
I=\frac{P}{A}
$$

Therefore, the energy density I opposite proportional to radius square (r2), as seen on;

$$
I=\frac{P}{4 \pi r^{2}}
$$

Comparing eq (28) and eq. (30) clear congruence the height of electric field and magnetic one, where less along the lines of

\section{Radiation Pressure}

Radiation pressure is the pressure exerted upon any surface exposed to electromagnetic (EM) radiation. Although radiation pressure can be understood using classical electrodynamics, here we will examine the quantum mechanical argument. From the perspective of quantum theory, light is made of photons: particles with zero mass but which carry energy and momentum. According to special relativity, because photons are devoid of mass, their energy (E) and momentum (p) are related by $E=p c$.

$$
\begin{aligned}
& b v_{d}=e E \\
& F_{B}=e F x B \\
& F_{B}=e v_{d} B=\frac{e^{2} E B}{b}=\frac{e^{2} E^{2}}{b c}
\end{aligned}
$$

Now consider a beam of light perpendicularly incident on a surface, and let us assume the beam of light is totally absorbed. The total energy rate absorbed by all target electrons found by;

$$
\frac{d U}{d t}=N \frac{d U_{E}}{d t}
$$

So the total force apply to the target is;

$$
F=N F_{B}=\frac{N e^{2} E^{2}}{b c}
$$

The momentum the photons carry is a conserved quantity (i.e., it cannot be destroyed) so it must be transferred to the surface; thus the absorption of the light beam causes the surface to gain momentum.

Second Law tells us that force equal's rate of change of momentum; thus during each second, the surface experiences a force (or pressure, as pressure is force per unit area) due to the momentum of the photons transfer to it. Recall eq. (25) the magnetic force, $\left(F_{B}\right)$ can be take the form of;

$$
\begin{aligned}
F_{B} & =\frac{1}{c} \frac{d U}{d t} \\
F_{B} & =\frac{S A}{c}
\end{aligned}
$$


$\frac{F_{a v}}{A}=\frac{I}{c}$

Eq. (38) gives us that, the pressure equal to momentum transferred per second per unit area equal to energy deposited per second per unit area.

\section{Maxwell Equation}

Therefore, if we add the displacement current to Ampere's Law, then we had the final equation form of Ampere's Law:[21-23]

$\nabla x H=J+\frac{d D}{d t}$

Multiply tow part of eq. (39) by curl at left one found;

$-\nabla E=-\mu \frac{d J}{d t}+-i \mu \frac{d^{2} D}{d t^{2}}$

As we know that, $D=\varepsilon_{0} E+P$, and $J=\sigma E$, so, eq. (40) become; [20]

$-\nabla E=-\mu \sigma \frac{d E}{d t}-i \mu \varepsilon_{0} \frac{d^{2} E}{d t^{2}}-\mu \frac{d P^{2}}{d t^{2}}$

$-\nabla E+\mu \sigma \frac{d E}{d t}+i \mu \varepsilon_{0} \frac{d^{2} E}{d t^{2}}=\mu \frac{d P^{2}}{d t^{2}}$

One can written eq. (42) in form;

$$
\begin{aligned}
& \frac{d^{2} E}{d z^{2}}=e^{i \theta}\left(\frac{d^{2} E_{m}}{d t^{2}}+-2 i k \frac{d E_{m}}{d t}-k^{2} E_{m}\right) \\
& \frac{d E}{d t}=\frac{\partial}{\partial t} \operatorname{Em}(z) e^{i(\omega t-k z)}=i \omega e^{i \theta} \\
& \frac{d^{2} E}{d t^{2}}=-\omega^{2} E_{m} e^{i \theta}
\end{aligned}
$$

Substitute's eqs. $(43,44,45)$ in eq. (42) and $P=\chi E$ one gets;

$-\frac{\partial^{2} E_{m}}{\partial z^{2}}+2 i k \frac{\partial E_{m}}{\partial z}+k^{2} E_{m}-\mu \sigma \omega E_{m}+i \mu \varepsilon_{0} \omega^{2} E_{m}=i \mu \chi \omega^{2} E_{m}$

Considering that $\frac{\partial^{2} E_{m}}{\partial z^{2}}<<\frac{\partial E_{m}}{\partial z}$ eq. (46) become;

$2 i k \frac{\partial E_{m}}{\partial z}=\left(k^{2}-\mu \sigma \omega\right) i E_{m}+\left(\mu \varepsilon_{0} \omega^{2}+i \mu \chi \omega^{2}\right) E_{m}$

Divided tow side of eq. (47) by (ik) then;

$$
\begin{aligned}
& \frac{\partial E_{m}}{\partial z}=\left(\frac{\mu \sigma \omega}{2 i k}-\frac{k}{2 i}\right) E_{m}+\left(\frac{\mu \varepsilon_{0} \omega^{2}+\mu \chi \omega^{2}}{2 k}\right) E_{m} \\
& \frac{\partial E_{m}}{E_{m}}=\left(\frac{\mu \sigma \omega}{2 i k}-\frac{k}{2 i}\right) \partial z+\left(\frac{\mu \varepsilon_{0} \omega^{2}+\mu \chi \omega^{2}}{2 k}\right) \partial z
\end{aligned}
$$

By integrated eq. (49) one found;

$$
\ln E_{m}=\left(\frac{\mu \sigma \omega}{2 i k}-\frac{k}{2 i}\right) z+\left(\frac{\varepsilon_{0}+\chi}{2 k}\right) \mu \omega^{2} z+C
$$

Where, $\mathrm{C}$ is content

$$
E_{m}=E_{0} e^{-i\left(\frac{\mu \sigma \omega}{2 k}-\frac{k}{2}\right) z}+e^{\left(\frac{\varepsilon_{0}+\chi}{2 k}\right) \mu \omega^{2} z}
$$


As specific angle between internal field and external one, in this case the susceptibility had to part real and imaginary as; $[20,22]$

$$
P=\chi E=\left(\chi_{1}+i \chi_{2}\right) E \Rightarrow \chi=\left(\chi_{1}+i \chi_{2}\right) \text {, this means that real part } \chi_{1} E_{\text {consider to parallel electric }}
$$

field done by the atom with external field, While, $\chi_{2} E$ consider to vertical electric field done by the atom with external field.

Therefore, eq. (51) take the form as;

$$
E_{m}=E_{0} e^{i\left(\frac{k}{2}-\frac{\mu \sigma \omega}{2 i k}\right) z}+e^{\left(\frac{\varepsilon_{0}+\chi_{1}+i \chi_{2}}{2 k}\right) \mu \omega^{2} z}
$$

Therefore, the field density got the form;

$$
E_{m}=E_{0} e^{\left(\frac{\varepsilon_{0}+\chi_{1}}{2 k}\right) \mu \omega^{2} z} e^{-i \widetilde{k} z} e^{i \omega t}
$$

From eq. (53), we found out the exchange information's of the field density, and what, happen to obtain that change. The first term of this equation, shows that the energy density exchange due to the number of bodies interact with radiation. This term lead to modification of amplitude of the wave, so, for a certain medium. One can gets, amplification of emission due to $\varepsilon_{0}, X_{1}$ of the number of interaction with the external field. Where, second and third term gave us the energy change depend to frequency and wave number.

Thus;

$$
\begin{aligned}
& \tilde{k}=\frac{k}{2}-\frac{\chi_{2} \mu \omega^{2}}{2 k}+\frac{\mu \sigma \omega}{2 k} \\
& E_{m}(z)=A_{0} e^{-i \tilde{k} z}
\end{aligned}
$$

To obtain the equation for lasing; $\tilde{k}=k$, so eq. 18 has the form;

$$
k^{2}=\mu \sigma \omega-\chi^{2} \mu \omega^{2}
$$

As we know that, $\frac{\omega}{k}=c \Rightarrow \omega=c k$, so eq, (56) had the form;

$$
k=\frac{\mu \sigma c}{1+\chi_{2} \mu c^{2}}
$$

The number of photons $\mathrm{N}$ proportional to the amplitude square of energy as;

$$
N \alpha A_{0}=E_{0}^{2} e^{2\left(\frac{\varepsilon_{0}+\chi_{1}}{2 k}\right) \mu \omega^{2} z}
$$

In case of $\mu=-|\mu|$, then eq. (17) become;

$$
\tilde{k}=\frac{2 \pi}{\tilde{\lambda}}=\frac{k}{2}+\frac{\chi_{2} \mu \omega^{2}}{2 k}-\frac{\mu \sigma \omega}{2 k}
$$

$$
\tilde{\lambda}=\frac{4 \pi}{k+\chi_{2} \mu c \omega-\mu \sigma c}
$$

From eq. (60) we found that the wavelength increase with conductivity increase and decrease with vertical field of atoms.

$$
\tilde{E}=h \tilde{v}=\frac{h v}{\tilde{\lambda}}=\frac{h c}{n \tilde{\lambda}}=\frac{\hbar c}{2 n}\left(k+\frac{\chi_{2} \mu \omega^{2}}{k}-\frac{\mu \sigma \omega}{k}\right)
$$

Thus, the energy decrease with conductivity increase.

At the conclusion of this research to be reference to the importance and strength of the Maxwell equations and their ability to boil, explain some of the phenomena and processes in nature. As demonstrated in the past the existence of electromagnetic waves in space and spread to two areas electrical and magnetic perpendicular and quickly move equal to the speed of light. Maxwell's equations will give a new information private from electromagnetic radiation interaction with the material. 


\section{Conclusions}

1. Since, the luminance is proportional to the number of photons, that means that the number of photons is proportional to the breadth of electromagnetic wave by the equation

2. Equation (58), notes that the number of photons produced from electron transition inside the atom depends on $\chi_{1}$ and thus, on the susceptibility. That express maize sense external domain that means the response of the outer-sphere with corn in the form of photons also appears in the form of absorption or emission of photons that shows the compatibility of quantum mechanics and the theory of Maxwell.

3. Moreover, it proved that the wavelength of the electromagnetic radiation decreases within the matter when pass through. Equation (61) found that the electromagnetic radiation energy decreased with increasing conductivity, which indicates that part of the radiation energy exhausted in the form of an electric current inside conductor.

4. Equation (58) is noted that, the conditions of occurrence of lasing requires that depends on the wavelength, permeability, and conductivity

\section{References}

[1]. U. Vogt, R. Frueke, T. Wilhein, H. Stollberg, P. A. C. Jansson, H. M. Hertz, Appl. Phys. B 78, 53-58, 2004.High-resolution Spatial Characterization of Laser Produced Plasmas at Soft X-ray Wavelength.

[2]. T. Dimire, E. T. Gumbrell, R. A. Smith, L. Mountford, and M. H. R. Hutchinson, , Phys. Rev. let. 498-501 1996.Supersonic Ionization Wave Driven by Radiation Transport in a Short-Pulse Laser-Produced Plasma

[3]. Jan Tuner and Lars Hode, , Prima Books AB 2002.Laser Therapy.

[4]. William T. Silfvast, , U.S.A 1999. Laser Fundamentals.

[5]. K Muraoka-M Maeda, P, 131, IOP. Publishing 1td, 2001.Laser-Aided Diagnostics of Cases and Plasmas

[6]. Tephen Jacobs, Murray Sargi, and Marlan O. Scally, High Energy Lasers and their Applications, Addison-Wesley Publishing Company Phys. of Quantum Electron, vol.1.

[7]. Sergey V. Fedorov, Nikolay N. Rosanov, Anatoly N. Shatsev, Nikolay A. Veretenov, and Andrei G. Vladimirov, IEEE JOURNAL OF QUANTUM ELECTRONICS, VOL. 39, NO. 2, FEBRUARY 2003.Topologically Multicharged and Multihumped Rotating Solitons in Wide-Aperture Lasers With a Saturable Absorber.

[8]. Tobia Carozzi, Anders Eriksson, Bengt Lundborg, Bo Thidé and Mattias Waldenvik, Copyright c 1997, 1998, 1999, 2000, 2001, 2002, 2003 and 2004 by Bo Thidé. electromagnetic field theory exercises, typeset in LATEX 2.,

[9]. A.H. Abdelrahman, K.M. Haroun, M. D. Abdella, J. Basic. Appl. Sci. Res., 3(11)143-149, 2013. Modification of Schrödinger Equation in a media,

[10]. John Wiley and Sons, 1999, X-rays from Laser Plasmas, British library.

[11]. David J. Griffiths, 1999, Introduction to Electrodynamics, 3rd edition, Prentice Hall.

[12]. M. E. Couprie et. al, Phys. Rev. (A) Vol. 44, 1991. Results and analysis of free-electron-laser oscillation in a high-energy storage ring,

[13]. C. E. Heiles and F. D. Drake, Icarus 2, 281-292, 1963. The Polarization and Intensity of Thermal Radiation from a Planetary Surface.

[14]. Shalom Eliezer, publishing, 2002. The Interaction of High Power Lasers with Plasmas, Institute of phys.

[15]. Yaduvir Singh, 2011, Electromagnetic Field Theory, Pearson Education India.

[16]. Ghana and Joy, New yourk:Micro-Hill Book Company,1972. An Introduction to modern optics.

[17]. O Hariharan and F Orlando, Academic press,1985. Optical Interferometry.

[18]. 2nd ed., New York: John Wiley and Sons, 1967. Geometrical and physical Optics.

[19]. D. Fleisch, Cambridge Univ. Press, $1^{\text {st }}$ ed. 2008.A students guide to Maxwell's equations.

[20]. A.H. Abdelrahman, M. D. Abdella, Mahgoub Salih, 1(4): 80-85 December 2012. The affect of External Electric Field on the Lasing Mechanism in the Fluid, Scientific Research and Impact.

[21]. D. Griffith, Benjamin Cummings, $3^{\text {rd }}$ ed. 1999.Introduction to Electrodynamics.

[22]. J. Adams Stratton, McGraw Hill, $1^{\text {st }}$ ed. 1941, Electromagnetic theory (Pure and Applied Physics.

[23]. J. D. Jackson, Wiley, $3^{\text {rd }}$ ed. 1998, Classical Electrodynamics,. 